\title{
Philippe Forest, Beaucoup de jours, d'après Ulysse de James Joyce
}

\section{Gabriella Bosco}

\section{Q OpenEdition \\ 1 Journals}

\section{Edizione digitale}

URL: https://journals.openedition.org/studifrancesi/4867

DOI: 10.4000/studifrancesi.4867

ISSN: 2421-5856

\section{Editore}

Rosenberg \& Sellier

\section{Edizione cartacea}

Data di pubblicazione: 1 avril 2012

Paginazione: 204-205

ISSN: 0039-2944

\section{Notizia bibliografica digitale}

Gabriella Bosco, «Philippe Forest, Beaucoup de jours, d'après Ulysse de James Joyce», Studi Francesi

[Online], 166 (I | LVI) | 2012, online dal 30 novembre 2015, consultato il 19 novembre 2021. URL: http:// journals.openedition.org/studifrancesi/4867 ; DOI: https://doi.org/10.4000/studifrancesi.4867

Questo documento è stato generato automaticamente il 19 novembre 2021.

\section{(c) (i) (9)}

Studi Francesi è distribuita con Licenza Creative Commons Attribuzione - Non commerciale - Non opere derivate 4.0 Internazionale. 


\title{
Philippe Forest, Beaucoup de jours, d'après Ulysse de James Joyce
}

\author{
Gabriella Bosco
}

\section{NOTIZIA}

PHILIPPE FOREST, Beaucoup de jours, d'après Ulysse de James Joyce, coll. «le livre la vie», Editions Nouvelles Cécile Defaut, Nantes, 2011, pp. 480.

1 Neonata collana delle raffinate Editions Nouvelles Cécile Defaut, «le livre la vie»affidata alle cure di Isabelle Grell - prende spunto da un progetto di libro cui Barthes accennò nel testo con il quale rifondò la scrittura autobiografica, Roland Barthes par Roland Barthes, esprimendo il proprio rammarico per non averla mai realizzata: «Le livre / la vie (prendre un livre classique et tout y rapporter de la vie pendant un an)». Il contratto editoriale della collana prevede dunque che un autore contemporaneo, scelta un'opera di un grande scrittore, filosofo, artista, abbia 365 giorni per trasformare in parole la sua relazione unica con quell'opera. Ovvero: che per un anno della sua vita si rapporti quotidianamente con l'opera scelta e faccia di questo intenso tu per tu un libro.

2 Una sorta di sfida lanciata da Barthes, raccolta a distanza di alcuni decenni da Cécile Defaut e Isabelle Grell, e messa nelle mani di alcuni tra i più interessanti narratori contemporanei. In Beaucoup de jours, Philippe FOREST fornisce la sua personale interpretazione del progetto del grande semiologo. Fa suo per un anno l'Ulisse di Joyce e lo dispiega per il lettore sviscerandone i meandri, esibendosi per 480 pagine in quello che è il suo esercizio stilistico preferito, nel quale potremmo dire che la sua scrittura s'identifica: il saggio narrativo, quello che si legge come un romanzo. È peraltro lo stesso Forest creatore di una narrativa che sempre più si apre alla teoria letteraria e ne diventa sede propositiva. In altri termini, l'A. fornisce oggi la più riuscita esecuzione di quella tierce forme che lo stesso Barthes inaugurò sfondando le frontiere di genere. 
3 La proposta insita nella collana «le livre la vie» è dunque quella di una lettura soggettiva del classico scelto, che testimoni del movimento di vasi comunicanti esistente per ognuno tra letteratura ed esistenza. L'Ulisse di Joyce, in particolare, è stato oggetto di tali e tanti commenti critici ed eruditi, che parlarne ancora non poteva passare, secondo l'A., se non per la formula molto personale autorizzata dal principio di questa collana. Allo stesso tempo, il saggio si trasforma in una sorta di guida alla lettura dell'Ulisse, finora inesistente in francese. Il titolo stesso, Beaucoup de jours, cita un passo del testo di Joyce, «A life is many days, day after day». Capitolo per capitolo, Forest fornisce una chiave interpretativa che rivela un testo molto più leggibile di quanto non lo si consideri abitualmente, e a sorpresa ne dimostra l'intensa umanità.

4 «Une journée qui compte pour toutes celles du calendrier: le 16 juin 1904. Un lieu qui vaut pour tous les lieux de la terre: Dublin. Une histoire qui comprend l'ensemble des fictions qui furent ou qui seront racontées: l'Odyssée d'Homère. Un héros qui est tout le monde puisque son nom est: Personne. [...] Un jour, une ville, une histoire, un héros: Personne vit les aventures de tout le monde en un lieu qui se situe nulle part comme il pourrait se trouver n'importe où, au cours de quelques heures aussi longues qu'un siècle et brèves autant qu'un instant» (dal Prologue, pp.13-14). A partire da questa premessa, il testo trascina il lettore in una navigazione che alterna onde verbali irte e scoscese a lente frasi di bellezza trasparente, riuscendo nell'impresa non facile di condurlo in porto, nel momento stesso in cui, finito l'anno di scrittura, l'A. mette il punto finale al saggio. 